\title{
Characterization of spatial memory reconsolidation
}

\author{
Xavier De Jaeger, ${ }^{1,2}$ Julie Courtey, ${ }^{1,2}$ Maïna Brus, ${ }^{1,2}$ Julien Artinian, ${ }^{1,2}$ Hélène Villain, ${ }^{1,2}$ \\ Elodie Bacquié, ${ }^{1,2}$ and Pascal Roullet ${ }^{1,2,3}$ \\ ${ }^{1}$ Université de Toulouse, Université Paul Sabatier, 31062 Toulouse Cedex 9, France; ${ }^{2}$ Centre de Recherches sur la Cognition Animale, \\ CNRS UMR 5169, 31062 Toulouse Cedex 9, France
}

\begin{abstract}
Reconsolidation is necessary for the restabilization of reactivated memory traces. However, experimental parameters have been suggested as boundary conditions for this process. Here we investigated the role of a spatial memory trace's age, strength, and update on the reconsolidation process in mice. We first found that protein synthesis is necessary for reconsolidation to occur in the hippocampal CA3 region after reactivation of partially acquired and old memories but not for strongly acquired and recent memories. We also demonstrated that the update of a previously stable memory required, again, a memory reconsolidation in the hippocampal CA3. Finally, we found that the reactivation of a strongly acquired memory requires an activation of the anterior cingulate cortex as soon as $24 \mathrm{~h}$ after acquisition. This study demonstrates the importance of the knowledge of the task on the dynamic nature of memory reconsolidation processing.
\end{abstract}

Retrieving a memory is not a passive phenomenon and can trigger different processes that can modulate stored information (Sara 2000; Suzuki et al. 2004). Indeed, the reactivation of a previously consolidated memory trace can render it labile again. If this memory reactivated trace is destabilized, in order to end this labile state, this trace must undergo a restabilization process called "reconsolidation" which requires new protein synthesis (Sara 2000; Nader 2003), notably for hippocampal-dependent memories (Debiec et al. 2002; Rossato et al. 2006; Artinian et al. 2007, 2008). Furthermore, the need for protein synthesis following memory reactivation has been found in different kinds of learning and memory using various tests such as fear conditioning (Nader et al. 2000), object recognition (Akirav and Maroun 2006), appetitive instrumental learning (Hernandez et al. 2002), eyelid conditioning responses (Inda et al. 2005), and spatial learning in the Morris water maze (MWM) (Rossato et al. 2006; Artinian et al. 2007, 2008).

Despite the widespread support for the general mechanism of reconsolidation, the fact that some studies have failed to block reconsolidation supports the idea that there are boundary conditions for the reconsolidation phenomenon (for details, see Dudai 2006; McKenzie and Eichenbaum 2011). First, memory age seems to be important and, over time, the memory trace seems to be less susceptible to disruption following its reactivation. This temporal gradient has been reported in very aversive associative tasks, such as passive avoidance and fear conditioning tasks (Milekic and Alberini 2002; Eisenberg and Dudai 2004; Suzuki et al. 2004), but also in other behavioral tasks, such as the conditioned place preference task (Robinson and Franklin 2010) and drug-associated operant conditioning (Lee et al. 2005). In these studies, the memory trace can be disrupted only a few days after its acquisition. However, in some cases this delay may be much larger, such as in contextual fear conditioning in which 45-d-old memories are always disrupted by protein synthesis after retrieval, showing that even a systems-consolidated memory undergoes hippocampal reconsolidation (Nader et al. 2000; Debiec et al. 2002).

The second important factor is memory strength. For example, in the conditioned place preference task, amnestic treatments disrupted reconsolidation for weak memories (four pairings), but

\footnotetext{
${ }^{3}$ Corresponding author

E-mail pascal.roullet@univ-tlse3.fr

Article is online at http://www.learnmem.org/cgi/doi/10.1101//m.033415.113.
}

had little effect on stronger memories (eight pairings) reactivated $1 \mathrm{~d}$ after training (Robinson and Franklin 2010). In the fear conditioning task, an increase of training strength by increasing the number of CS-US associations makes the reactivated memory trace less susceptible to interference (Suzuki et al. 2004; Lehmann and McNamara 2011). However, it has been reported that strong memories can still be destabilized and then undergo reconsolidation relatively to their age (Wang et al. 2009), suggesting that boundary conditions to the reconsolidation process can be in interaction.

The third factor is the incorporation of new information in the reactivated memory. Thus, one main function of reconsolidation is considered to be memory updating (Pedreira et al. 2004; Rossato et al. 2006). Even though Tronel et al. (2005) concluded that reconsolidation was not required to link new information to a retrieved memory, Lee (2008) demonstrated that an adaptive function of the reconsolidation process enables the update of memory's content. Without the presence of new information, thus when updating is not necessary, a very well-learned memory does not undergo reconsolidation (Rodriguez-Ortiz et al. 2005; Winters et al. 2009).

In most studies, investigators use associative aversive tests such as passive avoidance and, especially, the fear conditioning task. The use of these tests has allowed great progress in understanding the mechanisms and the role of reconsolidation. However, other behavioral paradigms would be better suited to study parts of the reconsolidation mechanisms. For example, studying memory update would bring new valuable information in a more complex form of learning such as spatial learning. Moreover, even if reconsolidation seems to be a relatively common process, some discrepancies persist and can be explained by differences in the type of memory measured or procedures used (Tronson and Taylor 2007). For these reasons, it seems crucial to test, in the same task, with the same line of animals, and certainly in the same lab, the boundary conditions for the reconsolidation phenomenon.

\footnotetext{
(C) 2014 De Jaeger et al. This article is distributed exclusively by Cold Spring Harbor Laboratory Press for the first 12 months after the full-issue publication date (see http://learnmem.cshlp.org/site/misc/terms.xhtml). After 12 months, it is available under a Creative Commons License (AttributionNonCommercial 4.0 International), as described at http://creativecommons. org/licenses/by-nc/4.0/.
} 
Furthermore, remote memories are suggested to be hippocampus-independent but rather dependent on the neocortex (Frankland et al. 2004). Therefore, the systems consolidation theory supports the idea that a gradual reorganization of the memory occurs over time where information originally encoded in the hippocampus is transferred to the cortical area (Frankland and Bontempi 2005). Recently, using a hippocampus-dependent task (contextual fear conditioning), Goshen et al. (2011) demonstrated that activation of the anterior cingulate cortex (aCC) is necessary during the recall of remote memories but not recent ones. However, in these experiments the cortical transfer occurred 3-4 wk after the initial acquisition. As we have already mentioned, a well-learned memory does not undergo reconsolidation unless in the presence of new information to update it (Rodriguez-Ortiz et al. 2005; Morris et al. 2006; Winters et al. 2009). However, the possible involvement of neocortical regions in the case of strong hippocampus-independent memories has never been explored in the case of spatial memory reconsolidation.

In the present study, we assessed the necessity of protein synthesis in the hippocampal CA3 area during the reconsolidation process (Artinian et al. 2007, 2008) according to the spatial memory trace's age, level of acquisition, and need for an update. Finally, we investigated whether the anterior cingulate cortex (aCC), a neocortical region known to process remote memories (Frankland et al. 2004), could replace the hippocampal CA3 in these conditions and would be the site of protein synthesis-dependent reconsolidation.

\section{Results and Discussion}

Morris et al. (2006) showed that inactivation of the dorsal hippocampus during reconsolidation by anisomycin (a protein synthesis inhibitor) has no effect on the MWM performances. However, in previous studies (Artinian et al. 2007, 2008), we showed that the hippocampal CA3 region is crucial for reconsolidation of spatial information in this same task. Different factors could explain this apparent discrepancy, but the most important fact is that, unlike us, Morris et al. (2006) used a distributed learning procedure leading to an asymptotic level of performances before the reactivation trial. Indeed, even if in our previous study mice had a good learning of the spatial task with the massed learning procedure, they did not reach an asymptotic level of performance.

In order to understand the importance of both factors (level of training before reactivation and distributed vs. massed procedure) in memory reconsolidation, we performed four parallel experiments with the same general conditions. First, we replicated the experiment in the Morris water maze with a simple massed acquisition procedure with reactivation (Fig. 1C; see Materials and Methods, Experiment 1; Artinian et al. 2008) or without reactivation (Fig. 1E; see Materials and Methods, Experiment 2 ). Then, we performed a distributed ac-
A

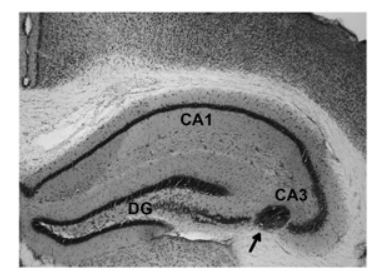

C
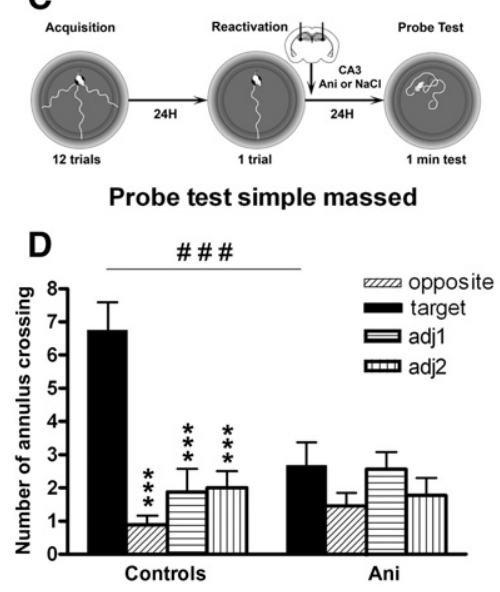

quisition procedure with only one learning trial per day for $12 \mathrm{~d}$ (Fig. 2A; see Materials and Methods, Experiment 3). In the fourth experiment, we doubled the number of trials in the massed procedure (Fig. 2C; see Materials and Methods, Experiment 4).

\section{Same level of acquisition with the different behavioral procedures}

As we can see in Figure 1B, during the first four sessions, the profile of acquisition and the level of final performances were the same whatever the procedure used $\left(F_{(3,99)}=0.641, P=0.590\right)$. Moreover, for the double massed acquisition procedure, during the last four sessions, there was no longer any significant evolution of the learning performances $\left(F_{(3,48)}=2.054, P=0.119\right)$, showing that the performances had reached an asymptotic level.

\section{Hippocampal CA3 region is crucial for spatial memory reconsolidation}

In the simple massed acquisition procedure (Fig. 1C), control animals searched for the platform at the exact location during the probe test occurring $24 \mathrm{~h}$ after the reactivation trial. By contrast, anisomycin (Ani) caused profound memory impairment in this massed procedure (Fig. 1D). A two-way ANOVA revealed no

Figure 1. Effects of intra-CA3 anisomycin infusion after reactivation (or not) following a single massed acquisition procedure. $(A)$ Representative sample of a thionine-stained brain section showing placement of the tip of the cannulae (black arrows). (DG) Dentate gyrus. (B) Throughout training sessions, mice learned equally well to locate the hidden platform and exhibited decreasing latencies over blocks of trials in the three different behavioral procedures (simple and double massed procedures, simple distributed procedure). (C) Experimental protocol for the single massed procedure. (D) Number of annulus crossings during probe tests. Control animals showed a preference for the target zone where the platform was located during training compared to the adjacent (Adj1 and Adj2) and opposite zones (target vs. others, $\left[{ }^{* * *}\right] P<0.001$ ) and there is no significant difference in the exploration of the four quadrants for the Ani group. Moreover, the control group crossed the target quadrant more often than the Ani group $\left(\left[{ }^{\# \# \#}\right] P<0.001\right)$. (E) Experimental protocol for the control of specific effects of anisomycin. $(F)$ Without the reactivation trial, $\mathrm{NaCl}$ - and Ani-injected mice crossed the annulus located in the target quadrant significantly more often than the remaining three annuli (target vs. others, $[* *] P<0.01,[* * *] P<0.001)$. 


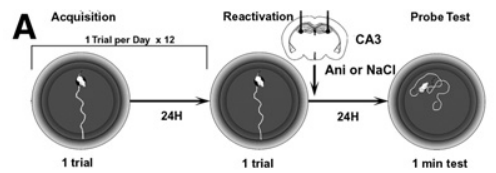

B Probe test simple distributed
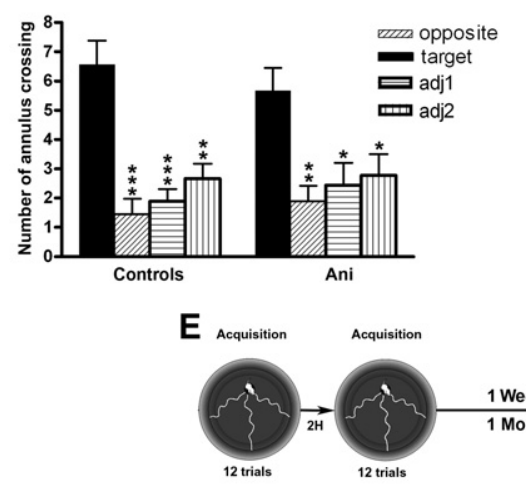

Probe test double massed 1 week

F

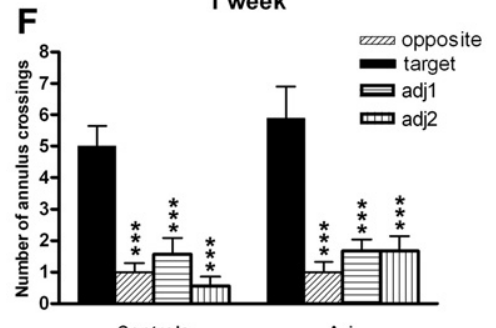

Controls

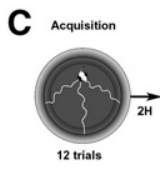

D

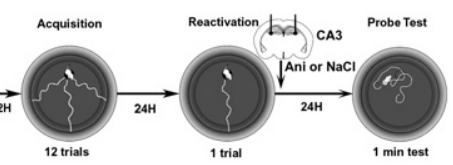

Probe test double massed

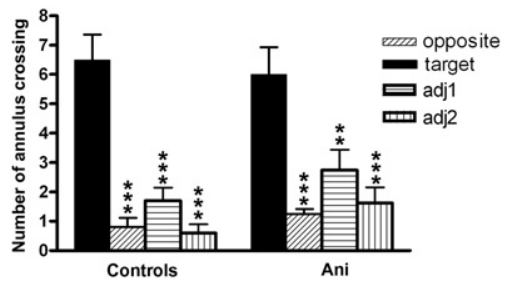

Figure 2. Effect of strength and age of the memory on the CA3 protein synthesis necessity during the reconsolidation. Experimental designs for the distributed procedure $(A)$ and for the double massed procedure $(C)$. Number of annulus crossings during probe tests in the distributed procedure $(B)$ and the double massed procedure $(D)$. All groups of mice showed a similar preference for the target zone where the platform was located during the training compared to the adjacent (Adj1 and Adj2) and opposite zones (target vs. others, $\left[{ }^{*}\right] P<0.05$, $\left.\left[{ }^{* *}\right] P<0.01,{ }^{* * *}\right] P<0.001$ ). (E) Experimental protocol used for the reactivation taking place $1 \mathrm{wk}$ or $1 \mathrm{mo}$ after the initial acquisition. Number of annulus crossings during probe tests occurring $1 \mathrm{wk}(F)$ or $1 \mathrm{mo}(G)$ after a double massed acquisition. One week after the acquisition, both groups of mice showed a similar preference for the target zone where the platform was located during training. However, for a 1-mo delay, there was no significant difference in the exploration of the four quadrants for the Ani group (target vs. others, $\left[{ }^{*}\right] P<0.05,\left[{ }^{* * *}\right] P<0.001$ ).

significant general effect between $\mathrm{NaCl}$ - and Ani-injected mice $\left(F_{(1,60)}=3.424, P=0.069\right)$, a significant quadrant effect $\left(F_{(3,60)}=\right.$ $13.939, \quad P<0.001)$, and a treatment $\times$ quadrant interaction $\left(F_{(3,60)}=7.420, P<0.001\right)$. Ani injection $24 \mathrm{~h}$ after initial acquisition and without reactivation did not impair long-term retention during the probe test performed $24 \mathrm{~h}$ later $\left(F_{(3,56)}=0.107\right.$, $P=0.956$ ) (Fig. 1F). Thus, without any reactivation trial, Ani injection had no effect on memory performances, showing that the memory deficit obtained in the first experiment was not due to a nonspecific effect of the drug and that the Ani effects are contingent on reactivation. Thus, we found again that the hippocampal CA3 region is crucial for reconsolidation of spatial information.

\section{Anisomycin did not impair spatial memory reconsolidation after distributed or double massed acquisition}

For the distributed procedure, during the probe test (Fig. 2A) both groups crossed the target annulus more often than the three other annuli $\left(F_{(3,64)}=18.915, P<0.001\right)$ indicating that mice remembered the location of the hidden platform (Fig. 2B). A two-way
ANOVA revealed no significant effect of the treatment $\left(F_{(1,64)}=0.014, P=0.905\right)$ and no interaction between the treatment and the exploration of the four annuli $\left(F_{(3,64)}=0.506, P=0.680\right)$. This indicates that Ani injection after reactivation does not produce a memory deficit after a distributed learning procedure where the number of trials and level of performances are identical to the simple massed procedure and only the number of reactivations has been increased.

During the probe test of the fourth experiment, with the double massed procedure (Fig. 2C), control and Ani-injected mice crossed the target annulus significantly more often than the remaining three annuli (Fig. 2D). The twoway ANOVA revealed a significant quadrant effect $\left(F_{(3,64)}=35.205, P<0.001\right)$, no treatment effect $\left(F_{(1,64)}=1.492, P=\right.$ 0.226 ), and no interaction between these two factors $\left(F_{(3,64)}=0.767, P=0.517\right)$. This result indicates that when the number of learning trials was increased, Ani injection after reactivation did not produce a memory deficit anymore. In addition, it may be noted that the level of performance is the same in the double acquisition procedure as in the simple acquisition procedure in control mice. Actually, without the platform the animal gives up and starts exploring other potential positions for the platform to escape even if they are well trained.

Together, results from this first set of experiments indicate that injection of anisomycin in CA3 produces amnesia for already consolidated spatial memory only when mice are submitted to a simple massed acquisition. If we increased the number of learning trials (Experiment 4) or the number of reactivations (Experiment 3), the memory trace becomes insensitive to the anisomycin injection during the reactivation. These results are in line with concluding remarks from Alberini's review (Alberini 2005) and the experiment from Winters et al. (2009) in which a longer exposition to the object prevents the memory destabilization after the reactivation trial. According to Alberini, "each training and reactivation event contributes to a gradient of stabilization that gradually increases and eventually results in a fully consolidated trace that is insensitive to disruption." Our hypothesis is that when an animal learns a new task, there is an increase of the level of performances at each session, but the performances are still not optimal. In this case, the animal has not perfectly integrated the task, and at each reactivation the animal keeps learning and has to add new information to the memory trace, and protein synthesis is still necessary. However, when performances, and therefore the knowledge of the task, are optimal, there is no need to incorporate new information and, therefore, protein synthesis during reconsolidation is no longer necessary.

Over time, when the animal is resubmitted to the behavioral test, it might not perfectly remember the task. In this case, animals certainly need to incorporate new information and protein synthesis is again required during reconsolidation. 
To test this hypothesis, we replicated the experiment with the double massed acquisition but we increased the delay between acquisition and reactivation from $24 \mathrm{~h}$ to $1 \mathrm{wk}$ or $1 \mathrm{mo}$ (Fig. 2E; see Matterial and Methods, Experiment 5).

\section{Anisomycin impaired spatial memory reconsolidation after double massed acquisition over time}

We compared the latency for mice to find the platform during the reactivation trial $24 \mathrm{~h}(14.2 \pm 3.2 \mathrm{sec}), 1 \mathrm{wk}(15.2 \pm 3.1 \mathrm{sec})$, or 1 mo $(17.1 \pm 3.5 \mathrm{sec})$ after the initial acquisition phase and found no significant difference in terms of performances $(24 \mathrm{~h}$ vs. $1 \mathrm{wk}, F_{(1,34)}=0.046, P=0.831 ; 24 \mathrm{~h}$ vs. $1 \mathrm{mo}, F_{(1,31)}=0.361$, $P=0.552$; data not shown). Thus, mice did not forget the location of the platform and kept a good memory of the task 1 wk and even 1 mo after a double massed acquisition.

During the probe test $1 \mathrm{wk}$ after acquisition (Fig. 2F), both groups of animals crossed the target annulus more often than the three other annuli $\left(F_{(3,64)}=30.535, P<0.001\right)$ indicating that mice remembered the exact location of the hidden platform. A two-way ANOVA revealed no significant effect of the treatment $\left(F_{(1,64)}=1.895, P=0.173\right)$ and no interaction between the treatment and the exploration of the four annuli $\left(F_{(3,64)}=0.523\right.$, $P=0.668)$.

However, for the 1-mo delay, Ani injection impaired longterm memory during the probe test (Fig. 2G). A two-way ANOVA revealed no significant general effect between $\mathrm{NaCl}$ - and Ani-injected mice $\left(F_{(1,52)}=0.001, P=0.999\right)$, a significant quadrant effect $\left(F_{(3,52)}=11.412, P<0.001\right)$, and a treatment $\times$ quadrant interaction $\left(F_{(3,52)}=3.034,<0.05\right)$.

This result shows that the animals became sensitive again to the injection of anisomycin only when the reactivation trial took place 1 mo after acquisition. Interestingly, at this time animals always have a good knowledge of the task since they obtained good performances during the reactivation trial. However, if the general knowledge of the task is still good, animals may not remember all the environmental cues and animals certainly need to incorporate new information.

The incorporation of new elements seems to be a key factor in the mechanisms of memory reconsolidation. In the Morris water maze task, generally, the update behavioral procedures consist in changing the spatial location of the platform every day (Morris et al. 2006). In this case, animals have to learn a new spatial location each day but always in the same spatially stable environment. This is a particular type of update because the animal must inhibit what he learned the day before, maybe implicating mechanisms of extinction and relearning.

However, updating memories is often very different and, to explain this, we will make an analogy with pathfinding in humans. When we move in a well-known city, to go to our favorite store, we know exactly the shortest way to access it. This is very similar to the standard procedure used in the Morris water maze. If arriving at the store we realize it is closed, we must find another equivalent store open in the city (similar to the update procedure conventionally used in the MWM). We must find another location in a stable environment. However, very often, we do exactly the opposite, i.e., we always go to the same place but in a changing spatial environment. Indeed, to continue the analogy, the store is always in the same place but some elements of the environment will be changed or deleted and others will appear (road works, construction or demolition of a house, facade renovations, etc.). This will change a part of the spatial environment and we must incorporate these new elements in our representation of the city, although these are not necessarily needed to find our store.

\section{Anisomycin impaired spatial memory reconsolidation after double massed acquisition when the memory is updated}

We wanted to develop a behavioral task that would involve the update of information from the environment while the spatial location of the platform would remain unchanged. To accomplish this, animals learned to locate the platform in the same stable environment just as in previous experiments with the double massed acquisition procedure (Fig. 3A). Under these experimental conditions, we know that injection of anisomycin during the reactivation has no effect on memory performances measured during the probe test (Fig. 2D). Next, four patterns were added to the spatial environment during the reactivation trial (Fig. 3B,C; see Materials and Methods, Experiment 6). Mice did not need these patterns to locate the platform since they had already learned the location using other elements, including the four first cues of the environment. Just after reactivation, animals were injected with anisomycin and tested $24 \mathrm{~h}$ later in the same environmental conditions as during reactivation.

During the reactivation trial with the four news patterns, mice are not disturbed as we can see from their excellent performances during the reactivation test (latency, $11.55 \pm 1.97 \mathrm{sec}$ ). These results are not different, and are perhaps a bit better, than in classical reactivation trials with the same acquisition conditions (latency, $14.22 \pm 3.15 \mathrm{sec}$ ).

During the probe test, an ANOVA test revealed no overall difference in the total number of crossings by Ani- or NaCl-injected mice $\left(F_{(1,64)}=0.219, P=0.642\right)$, but a significant quadrant effect $\left(F_{(3,64)}=25.338, P<0.001\right)$ (Fig. 3D) and, more importantly, an interaction effect $\left(F_{(3,64)}=5.826, P<0.001\right)$. These results demonstrate that when new information is present in the environment, the memory trace is again sensitive to protein synthesis inhibition. With that said, we observed a significant difference between target and opposite quadrants, suggesting that the deficit observed in this experiment is less severe than that in all the other experiments.

To demonstrate that this effect is specific to the memory reconsolidation when new elements are incorporated into the environment and not a nonspecific effect of adding new patterns or a nonspecific effect of the drug, an additional control experiment was performed. For that, mice were trained in the original environment, injected with anisomycin or $\mathrm{NaCl} 24 \mathrm{~h}$ later without reactivation, and tested $48 \mathrm{~h}$ after acquisition with the new patterns (Fig. 3E; see Materials and Methods, Experiment 7). In this condition, Ani injection without reactivation did not impair long-term retention during the probe test $\left(F_{(3,52)}=0.900, P=0.448\right)$ (Fig. $3 F)$. Therefore, when animals are trained in the original environment, and simply tested with the new patterns in the absence of any prior reactivation, the memory performances are not degraded. Taken together, these results demonstrated that the memory degradation observed after an updating during reactivation (Experiment 6) was not due to a new learning but was caused by the blockade of protein synthesis in memory reconsolidation. Our results are in agreement with previous observations regarding object recognition memory in which reconsolidation appears to be necessary when new information is incorporated (Rossato et al. 2007) or if the memory needs to be updated (Winters et al. 2009). An important point of the present experiment is that the new information is incorporated in the precedent memory trace even if it is not immediately necessary for the memory task and even if animals are on a performance plateau. Moreover, the deficit observed following anisomycin injection seems less severe than for a general reconsolidation, suggesting that the destabilization/restabilization for an update of memory could be of a milder degree than is the case for general reconsolidation of the memory trace. 
A
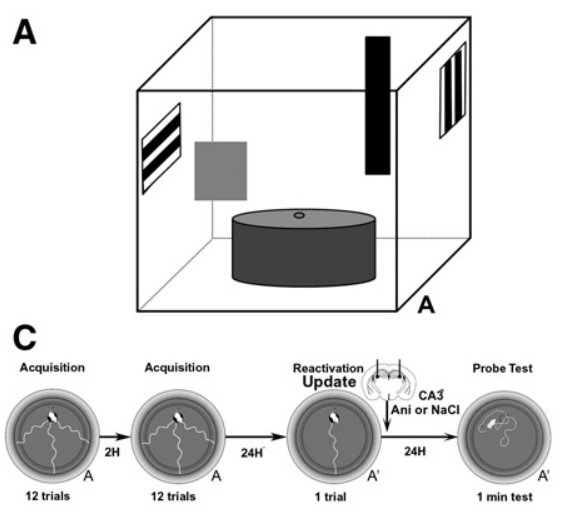

D

Probe test double massed with update

\#

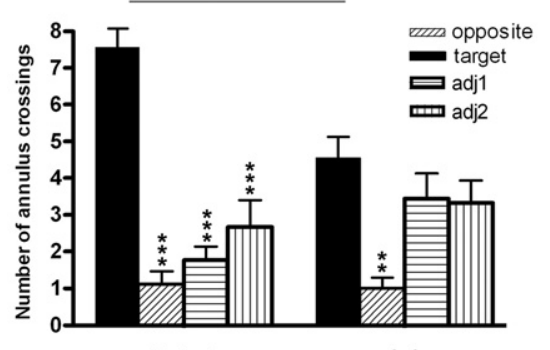

Controls

Ani

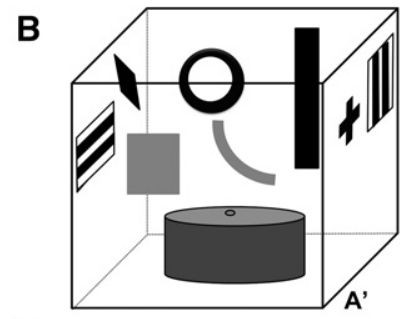

E

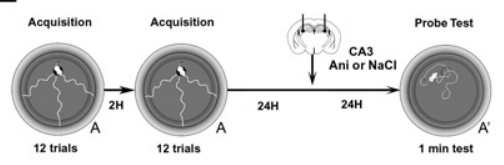

F Probe test double massed without reactivation

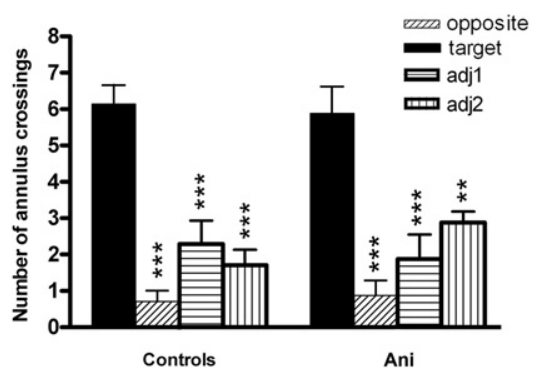

Controls

Ani

Figure 3. Role of protein synthesis in CA3 during an update of the memory. $(A)$ Schematic representation of the experimental spatial environments $(A)$ during acquisition. $(B)$ During the reactivation trial and the probe test, four new patterns have been added in the spatial environment $\left(A^{\prime}\right)$. (C) Experimental protocol used for the update of the memory. (D) Number of annulus crossings during probe tests. Control animals showed a preference for the target zone where the platform was located during training. For the Ani group, there was no significant difference in terms of exploration between the target and both adjacent quadrants. Moreover, the control group crossed the target quadrant more often than the Ani group. $(E)$ Experimental protocol used for the control of the specific effect of anisomycin for the update of memory procedure. $(F)$ Number of annulus crossings during probe test. Without the reactivation trial, $\mathrm{NaCl}$ - and Ani-injected mice crossed the annulus located in the target quadrant significantly more often than the remaining three annuli (target vs. others, $\left[{ }^{* *}\right] P<0.01$, $\left[{ }^{* * *}\right] P<0.001$; target quadrant controls vs. Ani group, $\left.\left[{ }^{\#}\right] P<0.05\right)$.

\section{aCC is engaged during the spatial memory recall after double massed acquisition}

It is also important to consider what happens during reactivation of a perfectly mastered task when there is nothing new to learn or add. The hippocampal CA3 region does not seem to be involved anymore in the reactivation of spatial information, and other brain regions should take over. The cortical linkage theory suggests that the hippocampus is gradually disengaged as cortical connections are strengthened (McKenzie and Eichenbaum 2011). It has been shown that the recall of remote memory displays an increase in the activation of the immediate early gene c-Fos in the aCC, and that an inactivation of this region during the recall of old contextual fear memories is able to alter the expression of this memory, whereas the hippocampus is only important for recent memory (Goshen et al. 2011). Similar hippocampal disengagement in favor of the aCC could be observed in our strong learning conditions. In order to test this hypothesis, we blocked CA3 or aCC specifically during the recall phase. For this, we changed the experimental procedure (Fig. 4A; see Materials and Methods, Experiment 8). We compared the effect of inactivation by lidocaine injection in the two structures in mice just before the probe test, after a simple massed learning (12 trials) or a strong massed learning (24 trials) of the spatial version of the Morris water maze. During the probe way ANOVA revealed no significant general effect between NaCl- and lidocaine-injected mice $\left(F_{(1,72)}=\right.$ $1.229, P=0.271)$, a significant quadrant effect $\left(F_{(3,72)}=19.009\right.$, $P<0.001)$, and a treatment $\times$ quadrant interaction $\left(F_{(3,72)}=\right.$ $8.037, P<0.001)$. This inactivation of aCC by lidocaine also increases the escape latency to cross, for the first time, a circle located at the original platform location (Latency: $\mathrm{NaCl}=6.15 \pm 2.52 \mathrm{sec}$; Lidocaine $\left.=19.2 \pm 5.8 \mathrm{sec} ; F_{(1,16)}=4.916, P=0.041\right)$. In conclusion for this section, the inactivation of the aCC during recall induced amnesia but only after a double massed acquisition.

These results first showed that the hippocampal CA3 region is crucial only for the recall of memories taking place $24 \mathrm{~h}$ after a weak acquisition. However, when the task is perfectly mastered, which is the case for the double massed learning, there is a disengagement of this hippocampal region during recall and no reconsolidation process is needed in this area after the reactivation. The crucial point of this study is that the aCC is involved during the reactivation, taking place $24 \mathrm{~h}$ after the initial acquisition, but only in the case of a perfectly mastered spatial task. Thus, after the disengagement of the hippocampal CA3 area, this cortical structure seems to take over. Now, the next step is to determine whether the reactivation after a strong learning renders the memory trace labile in the aCC, as is the case in the CA3 after a weak learning. Thus, to know whether this structure was involved in the process of memory reconsolidation of spatial information 
A

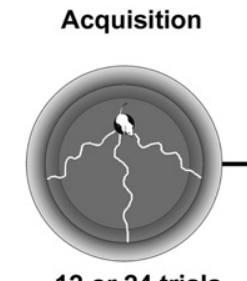

12 or 24 trials

CA3

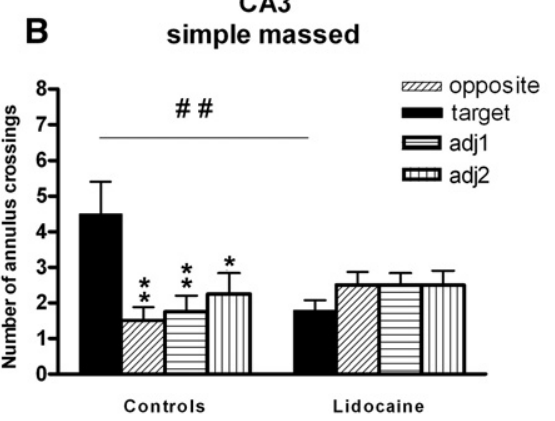

aCC

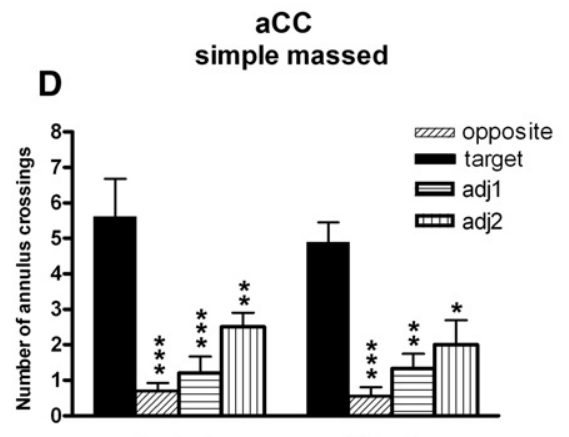

Controls

Lidocaine

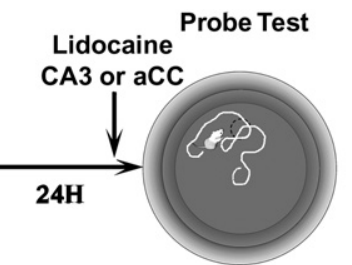

1 min test
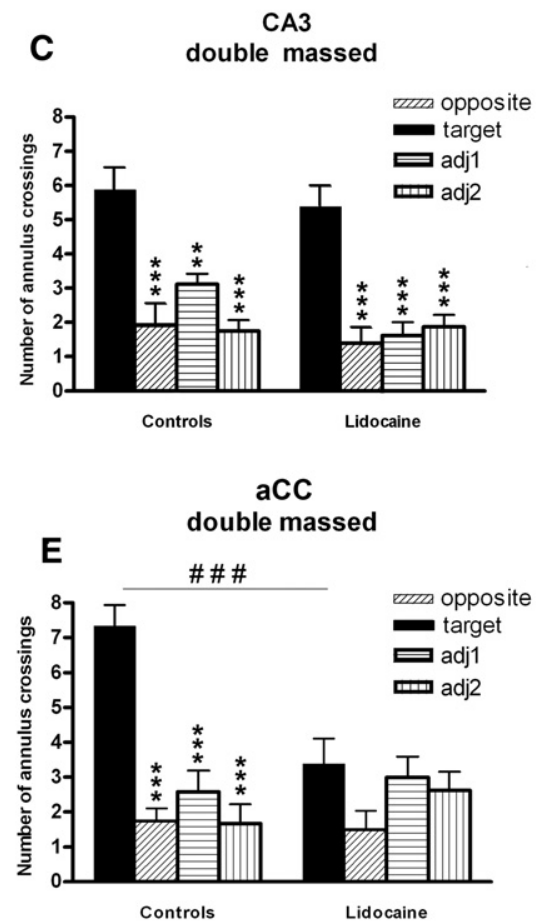

Figure 4. Inactivation of $\mathrm{CA} 3$ or aCC during recall after a simple or a double massed learning. $(A)$ Twenty-four hours after training, lidocaine or $\mathrm{NaCl}$ injections were performed just before the recall test in the CA3 hippocampal region or in the anterior cingulate cortex $(\mathrm{aCC})$. ( $B-E)$ Number of annulus crossings performances during probe tests. Lidocaine injection in the CA3 hippocampal region provoked a strong deficit during recall after a simple acquisition $(B)$ but not after a double acquisition $(C)$. On the contrary, the same injection of lidocaine in the aCC provoked amnesia only after a double massed acquisition $(D, E)$ (target vs. others, $\left[{ }^{*}\right] P<0.05,\left[{ }^{* *}\right] P<0.01,\left[{ }^{* * *}\right] P<0.001$; target quadrant controls vs. lidocaine group, $\left[{ }^{\# \#}\right] P<0.01$, $\left.\left.{ }^{\# \# \#}\right] P<0.001\right)$.

after a single or a double acquisition, we inhibited protein synthesis in the aCC by anisomycin injection after a reactivation trial in the Morris water maze (Fig. 5A; see Materials and Methods, Experiment 9).

During probe tests after a single or a double acquisition (Fig. 5B,C), saline- and Ani-treated mice demonstrated a strong preference for the target annulus where the platform was located during the acquisition task (single acquisition, $F_{(3,64)}=92.147$, $P<0.001$; double acquisition, $\left.F_{(3,56)}=27.929, P<0.001\right)$. With both procedures, no effect of Ani-treatment (single acquisition, $F_{(1,64)}=0.596, P=0.443$; double acquisition, $F_{(1,56)}=0.353, P=$ $0.555)$ and no interaction between the treatment $\times$ quadrant exploration (single acquisition, $F_{(3,64)}=0.986, P=0.405$; double acquisition, $\left.F_{(3,56)}=0.249, P=0.862\right)$ were found.

Injection of anisomycin in the aCC, even after a strong learning, did not cause amnesia in this task when animals were tested $48 \mathrm{~h}$ after reactivation. Thus, the aCC was strongly involved during the recall phase after a double acquisition, but protein synthesis, and therefore treatment of information in this structure, seemed not to be necessary for memory reconsolidation in this specific condition. However, we do not know whether this structure is im- plicated in old memory reconsolidation or in the updating condition. It is possible that strong memory cannot be destabilized by a single training trial. However, Einarsson and Nader (2012) showed that blockade of protein synthesis in aCC with anisomycin after retrieval of a contextual fear memory disrupted reconsolidation and impaired recent memory as well as remote memory. But in these experiments, inhibiting NMDAR-NR2B activity in aCC also disrupts acquisition of fear conditioning, demonstrating that this cortical structure is involved in all memory stages in this aversive task and not specifically in remote memory.

\section{Conclusion}

We present here three important findings about the reconsolidation of spatial memories. First, the protein synthesis reconsolidation-dependence is no longer necessary in the hippocampal CA3 area when memories are strong (asymptotic level of knowledge), suggesting that the strength of the memory is the key parameter for the need of the reconsolidation process. This result may explain some discrepancies described in previous studies about the age of the memory trace (Nader et al. 2000; Milekic and Alberini 2002; Eisenberg and Dudai 2004; Suzuki et al. 2004; Robinson and Franklin 2010) and this led us to draw a putative general model in which the strength of the memory could explain our results (Fig. 6A). In fact, the age of the memory but also the number of reactivation events tend to alter or improve the strength of the memory, respectively. On the other hand, when the trace is weak (i.e., the animal is still learning or perhaps it is starting to forget), the reactivation provides new information for the animal. To incorporate this new information, there are certainly two different but complementary mechanisms. At first, the reactivated memory undergoes a destabilization process whereby memory trace becomes labile (Nader 2003). During this lability, new information can be incorporated. Then restabilization of this modified memory trace occurs through memory reconsolidation mechanisms (Lee 2008, 2009).

The second important result is that the protein synthesis reconsolidation-dependence becomes necessary again in the hippocampal CA3 when new information needs to be incorporated into the original trace to update the memory even if all the previous environmental cues are still present (Fig. 6B). This raises the idea that reactivation may render the initial memory trace malleable to allow the introduction of new information (Lee 2009; Gisquet-Verrier and Riccio 2012). As a consequence, the reconsolidation in the CA3 region would be necessary again to restabilize this trace after the recall, including the update of the original memory (Nader and Einarsson 2010).

Finally, we showed that normal activity in the aCC, but not in the hippocampal CA3, is required for the recall of memory with asymptotic levels of knowledge but without the need to perform a protein synthesis reconsolidation-dependent process. These 


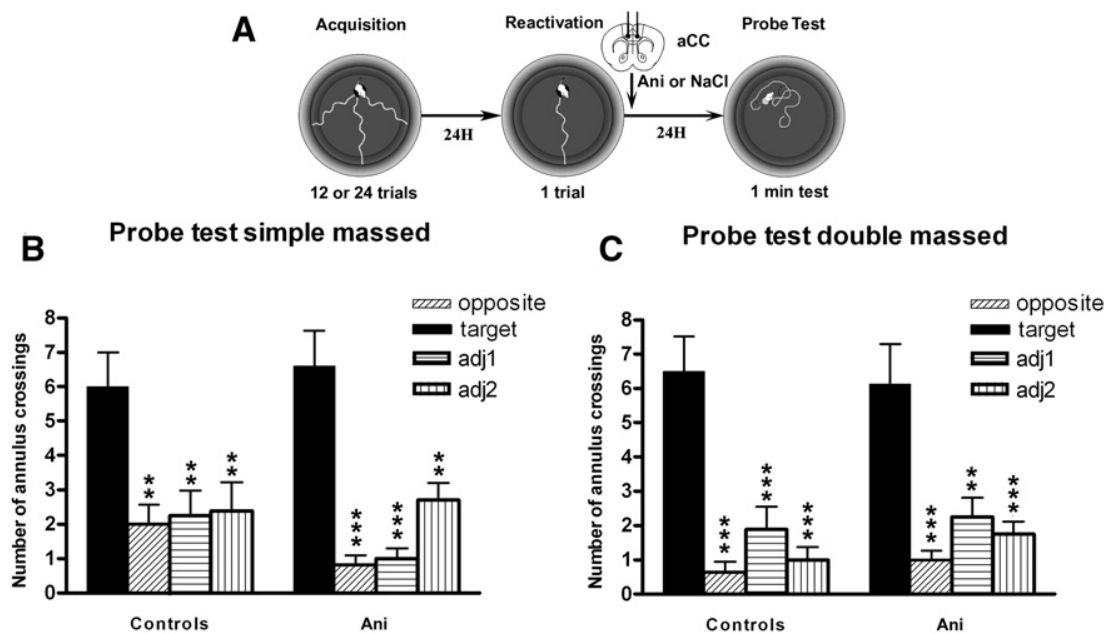

Figure 5. Protein synthesis inhibition in the aCC during reconsolidation. $(A)$ Twenty-four hours after a single or double acquisition procedure, a reactivation trial was performed and drug infusions in aCC occurred immediately after reactivation. The probe test took place $24 \mathrm{~h}$ after the injections. Number of annulus crossings during probe tests after a single $(B)$ and a double massed acquisition $(C)$. In both conditions, control mice and aCC Ani-injected mice during reconsolidation showed a similar preference for the target zone where the platform was located during training compared to the three other zones (target vs. others, $\left[{ }^{* *}\right] P<0.01,\left[{ }^{* * *}\right] P<0.001$ ).

findings are in accordance with the systems consolidation theory (Nader 2003) in which neocortical activation occurs during the retrieval of a 3- to 4 -wk-old remote memory (Bontempi et al. 1999; Frankland and Bontempi 2005). However, the neocortical engagement could occur much faster if an associative scheme into which new information is incorporated has previously been created (Tse et al. 2007). In the present study, we demonstrated that the aCC was required as soon as $24 \mathrm{~h}$ after a strong initial acquisition. Thus, neocortical involvement and, therefore, the dynamics of the memory trace depend not only on time but also on training strength, which is modulated by many factors including the number of learning/reactivating sessions and memory age.

\section{Materials and Methods}

\section{Experimental subjects}

A total of 242 (after histological control) CD1 male mice (IFFA CREDO, Lyon, France) were housed in groups of four in standard breeding cages placed in a rearing room at a constant temperature under diurnal conditions (12-h light-dark cycle [08:00-20:00]), with food and water ad libitum. At the time of the surgery, they were 100 -d old $( \pm 10 \mathrm{~d})$. They were tested during the first half of the light period. The strain of mice was chosen for their very good performances in spatial memory (Artinian et al. 2007, 2008). Moreover, CD1 mice exhibit a high endurance that is necessary in a massed procedure in the MWM.

\section{Ethics statement}

Every possible effort was made to minimize animal suffering and all experiments were performed in strict accordance with the recommendations of the European Union (86/609/EEC) and the French National Committee $(87 / 848)$.

\section{Surgery}

Bilateral guide cannulae $(0.56 \mathrm{~mm}$ in diameter) were implanted $1.2 \mathrm{~mm}$ above the CA3 region. The following coordinates with lambda and bregma in the same horizontal plane were used for the CA3 region: posterior to bregma, $-1.7 \mathrm{~mm}$; lateral to midline, $\pm 2.5 \mathrm{~mm}$, and $1.5 \mathrm{~mm}$ beneath the skull surface. For the aCC region, we used these coordinates: anterior to bregma, +0.9 $\mathrm{mm}$; lateral to midline, $0.5 \mathrm{~mm}$, and $1 \mathrm{~mm}$ beneath the skull surface. The subjects were then left in their home cage for a recovery period of $7-8 \mathrm{~d}$.

\section{Injection procedure}

Anisomycin (Ani- $\sigma)$, 4\% lidocaine hydrochloride (lidocaine- $\sigma$ ), or $\mathrm{NaCl}(0.9 \%)$ were bilaterally injected at a volume of $0.25 \mu \mathrm{L} /$ side into the dorsal hippocampus. Ani was initially dissolved in $3 \mathrm{~N}$ $\mathrm{HCl}$ and the solution was brought to a $\mathrm{pH}$ of 7.4 and to a final concentration of $100 \mu \mathrm{g} / \mu \mathrm{L}$ by addition of $3 \mathrm{~N} \mathrm{NaOH}$ and $\mathrm{NaCl}$. At this dose, Ani inhibits $>90 \%$ of protein synthesis in the brain during the first $2 \mathrm{~h}$ (Flood et al. 1973; Morris et al. 2006). To inactivate CA3 and aCC we used lidocaine, a sodium channel blocker that transiently suppresses neuronal firing. The injector (0.25 $\mathrm{mm}$ in diameter) was connected with a polyethylene tube to a $1-\mu \mathrm{L}$ Hamilton syringe driven by a microinjection pump at a rate of $0.1 \mu \mathrm{L} / \mathrm{min}$. Mice remained in their cages during the injection time. The injection lasted $2 \mathrm{~min} 30 \mathrm{sec}$ for each side and the needle was left in the cannulae for an additional $60 \mathrm{sec}$ to allow diffusion.
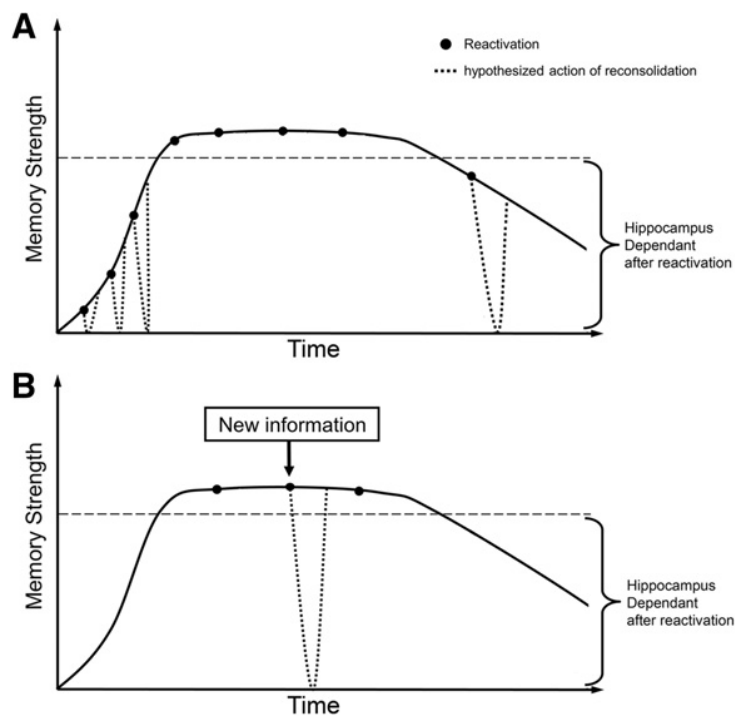

Figure 6. Schematic representation of memory reconsolidation. Black circles represent acquisition and reactivation trials and solid lines represent the observed behavioral performances. Dotted lines represent hypothesized actions of the reconsolidation. ( $A$ ) If a spatial task is not perfectly known, meaning that the animal is still learning (first part of the curve), the hippocampal protein synthesis is necessary after a reactivation, and a reconsolidation process is needed to keep building the memory trace. As soon as the performances, and therefore the knowledge of the task, are optimal (middle part of the curve), the trace is sufficiently established and, therefore, a protein synthesis reconsolidation dependence is no longer necessary in the hippocampus. Over time, when the animal certainly starts to forget information (end of the curve), the protein synthesis in the hippocampus is needed again for reconsolidation. (B) The incorporation of additional information in a well-established memory is able to destabilize the original memory trace even if this new information does not seem to be necessary for the resolution of the task. 


\section{Behavioral testing}

The apparatus and protocol for evaluating spatial memory were the same as in previous experiments (Florian and Roullet 2004). Briefly, mice were trained in the spatial Morris water maze (110 $\mathrm{cm}$ in diameter). A circular goal platform (9 $\mathrm{cm}$ in diameter) was placed in the center of one quadrant, $15 \mathrm{~cm}$ from the wall. Four start positions were located around the perimeter of the pool, dividing its surface into four equal quadrants. The apparatus was surmounted by a video camera connected to a video recorder and a computerized tracking system (Ethovision, Noldus).

Mice were individually submitted to a single familiarization session of three trials with the platform protruding $0.5 \mathrm{~cm}$ over the surface of the water. The majority of behavioral experiments (exceptions otherwise stated in the training phase) consisted of four consecutive sessions of three trials with an inter-session delay of 15-20 min during which mice were returned to their home cages. During the training phase, the platform was submerged $0.5 \mathrm{~cm}$ beneath the surface of the water. Mice were required to navigate to the invisible platform using the spatial cues available in the experimental environment. In each experiment, mice were tested for their long-term memory retention of spatial orientation by giving them a probe test consisting of a 60 -sec free swim trial in the absence of the platform.

\section{Experiment 1: simple massed acquisition procedure}

The training phase consisted of four consecutive sessions of three trials with an inter-session delay of 15-20 min (for a total time of $80 \mathrm{~min}$ ). Twenty-four hours after training, a reactivation trial consisting of an additional learning trial was performed. Mice were injected just after the reactivation trial and the probe test took place 24 h later.

\section{Experiment 2: control of specific effect of anisomycin}

In order to control the specific effect of anisomycin, a subset of mice received the same acquisition procedure as in Experiment 1 but did not undergo the reactivation trial and were merely left in their home cage. Injections were performed $24 \mathrm{~h}$ after the training sessions in the animal room and the probe test performed $24 \mathrm{~h}$ later.

\section{Experiment 3: distributed acquisition procedure}

Mice received one trial per day during $12 \mathrm{~d}$. Twenty-four hours after the last trial of acquisition, a reactivation trial was performed. Mice were injected just after the reactivation trial and the probe test took place $24 \mathrm{~h}$ later.

\section{Experiment 4: double massed acquisition procedure}

Two simple massed acquisition sessions identical to those in Experiment 1 were performed on the same day with a 2 -h interval between. A reactivation trial was performed $24 \mathrm{~h}$ after the training and mice were injected just after. The probe test was performed 24 $\mathrm{h}$ after the reactivation.

Experiment 5: reactivation $1 \mathrm{wk}$ or $1 \mathrm{mo}$ after the acquisition This experiment is similar to the fourth experiment with a reactivation $1 \mathrm{wk}$ or $1 \mathrm{mo}$ after the training.

Experiment 6: update of the memory during reactivation Here we follow the same protocol as in Experiment 4 but during the reactivation and the probe test, four new patterns were added in the spatial environment. Mice were injected just after the reactivation trial and the probe test took place $24 \mathrm{~h}$ later.

Experiment 7: update of memory; nonreactivation control In order to control the specific effect of anisomycin, a subset of mice received the same acquisition procedure as in Experiment
6 but did not undergo the reactivation trial and were merely left in their home cage. Injections were performed $24 \mathrm{~h}$ after the training sessions in the animal room and the probe test performed $24 \mathrm{~h}$ later with the four new patterns in the spatial environment.

\section{Experiment 8: inactivation of $\mathrm{CA} 3$ or aCC during} recall after a simple or a double massed learning Simple and double massed acquisition procedures were identical to those in Experiments 1 and 4, respectively. Twenty-four hours after the training, either a $\mathrm{NaCl}$ or a lidocaine injection was performed and, $8 \mathrm{~min}$ after, a 60 -sec recall test was performed.

\section{Experiment 9: protein synthesis inhibition in the aCC during reconsolidation}

Simple and double massed acquisition procedures were identical to those in Experiments 1 and 4, respectively. A reactivation trial was performed $24 \mathrm{~h}$ after the training and mice were injected in the aCC just after. The probe test was performed $24 \mathrm{~h}$ after reactivation.

\section{Behavioral variables}

All the behavioral groups were independent, i.e., each mouse was tested in one condition only. The number of mice by group was between eight and 12. For the training phase, mean escape latencies were recorded for each session (average of three trials per session). During the probe test, time spent in each quadrant and the number of annulus crossings, defined as the number of times a mouse crossed an ideal circle (14-cm diameter) located at the original platform location and the three equivalent areas in each of the other quadrants, was analyzed. We also measured the escape latency to cross for the first time the target annulus. The number of annulus crossings could reveal the strategy used while searching for the platform, while the number of target annulus crossings would determine whether mice had learned the target's location. Results for time in quadrants and annulus crossings were very similar and we have presented here only the number of crossings to avoid any redundancy in the data.

\section{Cannulae placement verification}

The cannulae positions were determined for each mouse by examination of serial coronal sections $(40 \mu \mathrm{m})$ stained with thionine (Fig. 1A). Only mice with both needle tracks terminating within the CA3 hippocampal region were included in the behavioral analysis.

\section{Statistical analysis}

SYSTAT 9.0 statistical software package was used for data analysis. The results were expressed as mean \pm SEM and analyzed using one- or two-way ANOVAS, or a repeated measure ANOVA when appropriate. Post-hoc multiple comparisons were carried out when allowed, using Tukey's Honestly Significant Distance (HSD) test.

\section{Acknowledgments}

This study was supported by CNRS ANR program (ANR-06Neuro-027-03) and by the University Paul Sabatier Toulouse 3. We thank A. Mele for the helpful discussions and A. La Fontaine and S. Hebert-Seropian for proofreading this manuscript.

\section{References}

Akirav I, Maroun M. 2006. Ventromedial prefrontal cortex is obligatory for consolidation and reconsolidation of object recognition memory. Cereb Cortex 16: 1759-1765.

Alberini CM. 2005. Mechanisms of memory stabilization: Are consolidation and reconsolidation similar or distinct processes? Trends Neurosci 28: 51-56. 
Artinian J, De Jaeger X, Fellini L, de Saint Blanquat P, Roullet P. 2007. Reactivation with a simple exposure to the experimental environment is sufficient to induce reconsolidation requiring protein synthesis in the hippocampal CA3 region in mice. Hippocampus 17: 181-191.

Artinian J, McGauran AM, De Jaeger X, Mouledous L, Frances B, Roullet P. 2008. Protein degradation, as with protein synthesis, is required during not only long-term spatial memory consolidation but also reconsolidation. Eur J Neurosci 27: 3009-3019.

Bontempi B, Laurent-Demir C, Destrade C, Jaffard R. 1999. Time-dependent reorganization of brain circuitry underlying long-term memory storage. Nature 400: 671-675.

Debiec J, LeDoux JE, Nader K. 2002. Cellular and systems reconsolidation in the hippocampus. Neuron 36: 527-538.

Dudai Y. 2006. Reconsolidation: the advantage of being refocused. Curr Opin Neurobiol 16: 174-178.

Einarsson EO, Nader K. 2012. Involvement of the anterior cingulate cortex in formation, consolidation, and reconsolidation of recent and remote contextual fear memory. Learn Mem 19: 449-452.

Eisenberg M, Dudai Y. 2004. Reconsolidation of fresh, remote, and extinguished fear memory in Medaka: old fears don't die. Eur J Neurosci 20: $3397-3403$.

Flood JF, Rosenzweig MR, Bennett EL, Orme AE. 1973. The influence of duration of protein synthesis inhibition on memory. Physiol Behav 10: $555-562$.

Florian C, Roullet P. 2004. Hippocampal CA3-region is crucial for acquisition and memory consolidation in Morris water maze task in mice. Behav Brain Res 154: 365-374.

Frankland PW, Bontempi B. 2005. The organization of recent and remote memories. Nat Rev Neurosci 6: 119-130.

Frankland PW, Bontempi B, Talton LE, Kaczmarek L, Silva AJ. 2004. The involvement of the anterior cingulate cortex in remote contextual fear memory. Science 304: 881-883.

Gisquet-Verrier P, Riccio DC. 2012. Memory reactivation effects independent of reconsolidation. Learn Mem 19: 401-409.

Goshen I, Brodsky M, Prakash R, Wallace J, Gradinaru V, Ramakrishnan C, Deisseroth K. 2011. Dynamics of retrieval strategies for remote memories. Cell 147: 678-689.

Hernandez PJ, Sadeghian K, Kelley AE. 2002. Early consolidation of instrumental learning requires protein synthesis in the nucleus accumbens. Nat Neurosci 5: 1327-1331.

Inda MC, Delgado-Garcia JM, Carrion AM. 2005. Acquisition, consolidation, reconsolidation, and extinction of eyelid conditioning responses require de novo protein synthesis. J Neurosci 25: 2070-2080.

Lee JL. 2008. Memory reconsolidation mediates the strengthening of memories by additional learning. Nat Neurosci 11: 1264-1266.

Lee JL. 2009. Reconsolidation: maintaining memory relevance. Trends Neurosci 32: $413-420$.

Lee JL, Di Ciano P, Thomas KL, Everitt BJ. 2005. Disrupting reconsolidation of drug memories reduces cocaine-seeking behavior. Neuron 47: $795-801$.

Lehmann H, McNamara KC. 2011. Repeatedly reactivated memories become more resistant to hippocampal damage. Learn Mem 18: $132-135$.
McKenzie S, Eichenbaum H. 2011. Consolidation and reconsolidation: two lives of memories? Neuron 71: 224-233.

Milekic MH, Alberini CM. 2002. Temporally graded requirement for protein synthesis following memory reactivation. Neuron 36: 521-525.

Morris RG, Inglis J, Ainge JA, Olverman HJ, Tulloch J, Dudai Y, Kelly PA. 2006. Memory reconsolidation: sensitivity of spatial memory to inhibition of protein synthesis in dorsal hippocampus during encoding and retrieval. Neuron 50: 479-489.

Nader K. 2003. Memory traces unbound. Trends Neurosci 26: 65-72.

Nader K, Einarsson EO. 2010. Memory reconsolidation: an update. Ann N Y Acad Sci 1191: 27-41.

Nader K, Schafe GE, Le Doux JE. 2000. Fear memories require protein synthesis in the amygdala for reconsolidation after retrieval. Nature 406: $722-726$.

Pedreira ME, Perez-Cuesta LM, Maldonado H. 2004. Mismatch between what is expected and what actually occurs triggers memory reconsolidation or extinction. Learn Mem 11: 579-585.

Robinson MJ, Franklin KB. 2010. Reconsolidation of a morphine place preference: impact of the strength and age of memory on disruption by propranolol and midazolam. Behav Brain Res 213: 201-207.

Rodriguez-Ortiz CJ, De la Cruz V, Gutierrez R, Bermudez-Rattoni F. 2005 Protein synthesis underlies post-retrieval memory consolidation to a restricted degree only when updated information is obtained. Learn Mem 12: $533-537$.

Rossato JI, Bevilaqua LR, Medina JH, Izquierdo I, Cammarota M. 2006. Retrieval induces hippocampal-dependent reconsolidation of spatial memory. Learn Mem 13: 431-440.

Rossato JI, Bevilaqua LR, Myskiw JC, Medina JH, Izquierdo I, Cammarota M. 2007. On the role of hippocampal protein synthesis in the consolidation and reconsolidation of object recognition memory. Learn Mem 14: 36-46.

Sara SJ. 2000. Retrieval and reconsolidation: toward a neurobiology of remembering. Learn Mem 7: 73-84.

Suzuki A, Josselyn SA, Frankland PW, Masushige S, Silva AJ, Kida S. 2004. Memory reconsolidation and extinction have distinct temporal and biochemical signatures. J Neurosci 24: 4787-4795.

Tronel S, Milekic MH, Alberini CM. 2005. Linking new information to a reactivated memory requires consolidation and not reconsolidation mechanisms. PLoS Biol 3: e293.

Tronson NC, Taylor JR. 2007. Molecular mechanisms of memory reconsolidation. Nat Rev Neurosci 8: 262-275.

Tse D, Langston RF, Kakeyama M, Bethus I, Spooner PA, Wood ER, Witter MP, Morris RG. 2007. Schemas and memory consolidation. Science 316: 76-82.

Wang SH, de Oliveira Alvares L, Nader K. 2009. Cellular and systems mechanisms of memory strength as a constraint on auditory fear reconsolidation. Nat Neurosci 12: 905-912.

Winters BD, Tucci MC, DaCosta-Furtado M. 2009. Older and stronger object memories are selectively destabilized by reactivation in the presence of new information. Learn Mem 16: 545-553.

Received September 30, 2013; accepted in revised form March 14, 2014. 


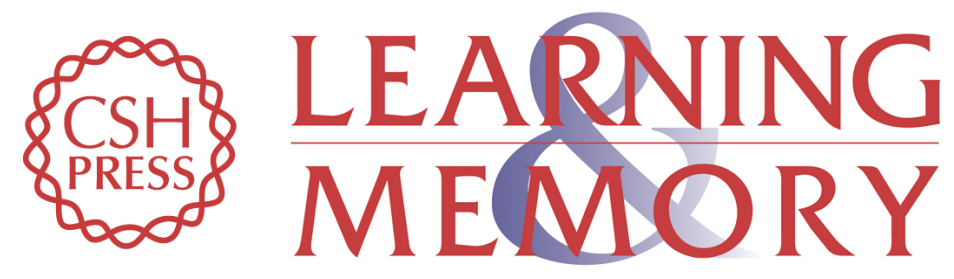

\section{Characterization of spatial memory reconsolidation}

Xavier De Jaeger, Julie Courtey, Maïna Brus, et al.

Learn. Mem. 2014, 21:

Access the most recent version at doi:10.1101//m.033415.113

References This article cites 39 articles, 13 of which can be accessed free at: http://learnmem.cshlp.org/content/21/6/316.full.html\#ref-list-1

Creative This article is distributed exclusively by Cold Spring Harbor Laboratory Press for the Commons first 12 months after the full-issue publication date (see

License http://learnmem.cshlp.org/site/misc/terms.xhtml). After 12 months, it is available under a Creative Commons License (Attribution-NonCommercial 4.0 International), as described at http://creativecommons.org/licenses/by-nc/4.0/.

Email Alerting Receive free email alerts when new articles cite this article - sign up in the box at the Service top right corner of the article or click here. 